\title{
LINEAR PICARD SETS FOR ENTIRE FUNCTIONS
}

\author{
SAKARI TOPPILA
}

\section{Introduction and results}

1. In the terminology of Lehto ([5]), a plane set $E$ is a Picard set for entire functions if every transcendental entire function takes all finite values with at most one exception infinitely often in the complement of $E$. It is proved in [8] that a countable set $E=\left\{a_{n}\right\}$ whose points converge to infinity is a Picard set for entire functions if there exists $\varepsilon>0$ such that

$$
\left\{z: 0<\left|z-a_{n}\right|<\frac{\varepsilon\left|a_{n}\right|}{\log \left|a_{n}\right|}\right\} \cap E=\phi
$$

for all large $n$. This result is sharp in the sense that, corresponding to each real-valued function $h(r)$ satisfying the condition $h(r) \rightarrow \infty$ as $r \rightarrow \infty$, there exists $E=\left\{a_{n}\right\}$ with $\lim a_{n}=\infty$ such that $E$ is not a Picard set for entire functions and

$$
\left\{z: 0<\left|z-a_{n}\right|<\frac{\left|a_{n}\right|}{h\left(\left|a_{n}\right|\right) \log \left|a_{n}\right|}\right\} \cap E=\phi
$$

for all large $n$. We shall prove the corresponding result for linear sets.

Theorem 1. If the positive numbers $e<a_{1}<a_{2}<\ldots$ satisfy the condition

$$
a_{n+1} \geq a_{n}+\frac{\varepsilon a_{n}}{\left(\log a_{n}\right)^{2}}
$$

for some $\varepsilon>0$ and for all large $n$, then $E=\left\{a_{n}\right\}$ is a Picard set for entire functions.

This theorem is sharp. It is proved in [9] that if $h(r) \rightarrow \infty$ as $r \rightarrow \infty$, there exist real numbers $0 \leq a_{1}<a_{2}<\ldots$ such that $E=\left\{a_{n}\right\}$ is not a Picard set for entire functions and

$$
a_{n+1} \geq a_{n}+\frac{a_{n}}{h\left(a_{n}\right)\left(\log a_{n}\right)^{2}}
$$

for all sufficiently large $n$. 
We denote by $U(z, r)$ the open disc of centre $z$ and radius $r$. We shall prove the following theorem from which Theorem I immediately follows.

Theorem 2. Let $0<\alpha \leq 2$. Let the positive numbers $e<a_{1} \leq a_{2} \leq \ldots$ satisfy the condition

$$
a_{n+1} \geq a_{n}+\frac{\varepsilon a_{n}}{\left(\log a_{n}\right)^{\alpha}}
$$

for some $\varepsilon>0$ and for all large $n$. If the radii of the discs $C_{n}=U\left(a_{n}, r_{n}\right)$ satisfy the condition

$$
\log \left(1 / r_{n}\right) \geq K\left(\log a_{n}\right)^{2+\alpha},
$$

where $K=20(1+1 / \varepsilon)$, then the union $\cup C_{n}$ is a Picard set for entire functions.

We denote by $[a, b]$ the closed segment $a \leq x \leq b$, and $(a, b)$ is the segment $a<x<b$. Theorem 2 is sharp in the following sense.

Theorem 3. Let $0<\alpha \leq 2$. There exists a countable set $E=\left\{a_{n}\right\}$, $0 \leq a_{1}<a_{2}<\ldots, \lim a_{n}=\infty$, satisfying (a) for $\varepsilon=1 / 7$ such that the linear set

where

$$
\bigcup_{n=1}^{\infty}\left(a_{n}-r_{n}, a_{n}+r_{n}\right)
$$

$$
\log \left(1 / r_{n}\right)=\frac{1}{8}\left(\log a_{n}\right)^{2+a}
$$

is not a Picard set for entire functions.

The corresponding sharp result for discs $C_{n}$ whose middle points need not lie on a ray is proved in [10]. If we have $0<\alpha \leq 1, \varepsilon>0$, and $K_{0}$ a sufficiently large constant, then the conditions

$$
\left\{z: 0<\left|z-a_{n}\right|<\frac{\varepsilon\left|a_{n}\right|}{\left(\log \left|a_{n}\right|\right)^{a}}\right\} \cap E=\phi
$$

and

$$
\log \left(1 / r_{n}\right) \geq K_{0}\left(\log a_{n}\right)^{2+2 \alpha}
$$

guarantee that $\bigcup_{n=1}^{\infty} U\left(a_{n}, r_{n}\right)$ is a Picard set for entire functions. If $K_{0}$ in (B) is taken too small, then $U U\left(a_{n}, r_{n}\right)$ need not be a Picard set.

2. Lehto and Virtanen [7] gave the following definition for normal meromorphic functions: If $f$ is meromorphic in a simply connected domain $G$, then $f$ is normal if and only if the family $\{f(S(z))\}$, where $\zeta=S(z)$ 
denotes an arbitrary one-to-one conformal mapping onto itself, is normal in the sense of Montel. In multiply connected domains $f$ is said to be normal if it is normal on the universal covering surface. We shall consider the following problem: If $E$ is a closed set, under what conditions does there exist a normal meromorphic function in the complement of $E$ with at least one essential singularity in $E$ ? Lehto and Virtanen [7] proved that if $f$ is normal in $G$, then $f$ is normal in every subdomain of $G$, and that a meromorphic function can not be normal in any neighbourhood of its isolated essential singularity. This implies that if $E$ is a finite set and $f$ is normal in $-E$, then $f$ is a rational function. Only finite sets have this property. We shall prove

Theorem 4. If $E$ is an infinite closed set, there exists a non-rational normal meromorphic function in $-E$.

We denote

$$
\varrho(f(z))=\frac{\left|f^{\prime}(z)\right|}{1+|f(z)|^{2}}
$$

The proof of Theorem 4 is based on the following theorem of Lehto and Virtanen [7].

Theorem A. A non-constant $f$, meromorphic in a domain $G$ of hyperbolic type, is normal in $G$ if and only if there exists a finite constant $C$ so that for all $z$ in $G$,

$$
\varrho(f(z))|d z| \leq C d \sigma_{G}(z),
$$

where $d \sigma_{G}(z)$ denotes the element of length in the hyperbolic metric of $G$.

3. We denote $S(\alpha, \beta)=\{z: \alpha \leq \arg z \leq \beta\}$ and $L_{\alpha}=L(\alpha)=$ $\{z: \arg z=\alpha\}$. Let $f$ be an entire transcendental function. We say that $L_{\alpha}$ is a line of Julia of $f$ if, for every $\varepsilon>0, f$ takes every finite value except perhaps one infinitely often in $S(\alpha-\varepsilon, \alpha+\varepsilon)$. The set of all Julia lines of $f$ is denoted by $J_{f}$. Then $J_{f} \neq \phi$ and the set $\left\{e^{i \alpha}\right.$ : $\left.L_{\alpha} \in J_{f}\right\}$ is closed. On the other hand, if $J=\left\{L_{\alpha}: \alpha \in E\right\} \neq \phi$, $\left\{e^{i \alpha}: \alpha \in E\right\}$ is closed, and $0 \leq \varrho \leq 1 / 2$ or $\varrho=\infty$, there exists an entire function $f$ of order $\varrho$ such that $J_{f}=J$. This is proved by Polya in the case $\varrho=\infty$ and by Anderson and Clunie [1] in the case $\varrho=0$. In fact, the function $f$ constructed by Anderson and Clunie can be chosen such that $f$ is slowly growing, ise.

$$
\log M(r, f)=O\left((\log r)^{2}\right)
$$

as $r \rightarrow \infty$. In the case $0<\varrho \leq 1 / 2$, we take $L_{\alpha} \in J$ and construct an entire function $g$ of order $\varrho$ such that, for every $\varepsilon>0,|g(z)|$ tends to infinity uniformly outside $S(\alpha-\varepsilon, \alpha+\varepsilon)$ as $|z| \rightarrow \infty$. Let $h$ be a slowly 
growing entire function such that $J_{h}=J$. Then $f(z)=g(z) h(z)$ is of order $\varrho$ and $J_{f}=J$.

Let now $f$ be an entire function of order $\varrho, 1 / 2<\varrho<\infty$. Cartwright [3] has proved that there exists $S(\alpha, \beta)$ where $\beta=\alpha+\pi / \varrho$, such that if $S(\gamma, \gamma+\pi / \varrho) \cap S(\alpha, \beta) \neq \phi$, then $S(\gamma, \gamma+\pi / \varrho)$ contains at least one Julia line of $f$. Therefore, if $1 / 2<\varrho<1$, there exist $L_{a}, L_{\beta} \in J_{f}$ such that $2 \pi-\pi / \varrho \leq \beta-\alpha \leq \pi$, and if $1 \leq \varrho<\infty$, there exist $L_{\alpha}, L_{\beta}$, $L_{\gamma} \in J_{f}$ such that $\alpha<\beta \leq \gamma, \beta-\alpha \leq \pi / \varrho, \gamma-\beta \leq \pi / \varrho$ and $\gamma-\alpha \geq \pi / \varrho$. Conversely, if $J$ satisfies these conditions, there exists an entire function of order $\varrho$ such that $J_{f} \subset J$. We shall prove

Theorem 5. Let $\left\{e^{i a}: \alpha \in E\right\}$ be closed and $J=\left\{L_{\alpha}: \alpha \in E\right\}$ $\neq \phi . I f$

(i) $1 / 2<\varrho<1$ and there exist $L_{\alpha}, L_{\beta} \in J$ such that $2 \pi-\pi / \varrho \leq$ $\beta-\alpha \leq \pi$ or

(ii) $1 \leq \varrho<\infty$ and there exist $L_{a}, L_{\beta}, L_{\gamma} \in J$ such that $\alpha<\beta \leq \gamma$, $\beta-\alpha \leq \pi / \varrho, \gamma-\beta \leq \pi / \varrho$ and $\gamma-\alpha \geq \pi / \varrho$,

then there exists an entire fuction $f$ of order $\varrho$ such that $J_{f}=J$.

We denote $\log ^{+} d=\max \{0, \log d\}$ for $d \geq 0$. We need Schottky's theorem in our considerations. It is proved by Ahlfors in the following form.

Schottky's theorem. If $g$ is regular and $g(z) \neq 0,1$ in $|z|<1$, then

$$
\log ^{+}|g(z)| \leq \frac{1+|z|}{1-|z|}\left(7+\log ^{+}|g(0)|\right) \text {. }
$$

\section{Proof of Theorem 2}

4. Contrary to our assertion, let us suppose that there exist an entire transcendental function $f$ and $r_{0}>0$ such that

$$
f^{-1}(\{0,1\}) \subset U\left(0, r_{0}\right) \cup \bigcup_{n=1}^{\infty} C_{n} .
$$

It does not mean any restriction to assume that $f\left(C_{n}\right) \cap\{0,1\} \neq \phi$ for every $n$. Similarly, we may assume that $f(0) \neq 0$, because if $f(0)=0$ then we can consider the function $1-f$. We denote by $K_{1}, K_{2}, \ldots$ constants depending only on the numbers $\varepsilon$ and $\alpha$ in Theorem 2, and $M_{1}, M_{2}, \ldots$ are constants depending on $f$.

Let $r>2 r_{0}$. Applying Schottky's theorem to the function $h(z)=$ $f\left(z^{2}\right)$ we see that $\log ^{+}\left|h\left(i r^{\frac{1}{2}}\right)\right| \leq M_{1} r^{\frac{1}{2}}$ and therefore $\log ^{+}|f(-r)| \leq M_{1} r^{\frac{2}{2}}$. Applying Schottky's theorem repeatedly, we see that $\log ^{+}|f(z)| \leq M_{2} r^{\frac{1}{2}}$ on 


$$
\{-r+i y:|y| \leq r\} \cup\{x+i y:|y|=r, \quad-r \leq x \leq 2 r\} .
$$

It follows from (a) and (b) that we can choose $s, r \leq s \leq 2 r$, such that, for any $n, U(s, \delta) \cap C_{n}=\phi$ where

$$
\delta=\frac{\varepsilon r}{4(\log r)^{\alpha}} .
$$

Applying Schottky's theorem in $U(s+i r, r-\delta / 4)$ and in $U(s-i r$, $r-\delta / 4)$, we see that

$$
\log ^{+}|f(s+i y)| \leq M_{3}(\log r)^{a} r^{\frac{1}{2}} ;
$$

if $\delta / 2 \leq|y| \leq r$, and the same theorem applied in $U(s, \delta)$ gives

$$
\log ^{+}|f(s+i y)| \leq M_{4}(\log r)^{\alpha} r^{\frac{1}{2}}
$$

for $-r \leq y \leq r$. Now it follows from the maximum principle that $\log M(r) \leq M_{4}(\log r)^{a} r^{\frac{1}{2}} ;$, where $M(r)=M(r, f)=\max \{|f(z)|:|z|=$ $r\}$. The order of $f$ is at most $1 / 2$ and we may write

$$
f(z)=b \prod_{\nu=1}^{\infty}\left(1-z / t_{\nu}\right)
$$

where $0<\left|t_{1}\right| \leq\left|t_{2}\right| \leq \ldots$ and $b \neq 0$.

5. Let us suppose that there exists $n_{0}$ such that $\{0,1\} \subset f\left(C_{n}\right)$ for every $n \geq n_{0}$. Let $r>10 a_{n_{0}}$. If either $a_{n} \leq 3 r / 4-1$ or $a_{n} \geq r+1$ for any $n$, then $n(r)=n(3 r / 4)$, where $n(t)=n(t, 0)$ is the number of zeros of $f$ in $|z| \leq t$, zeros of order $p$ being counted $p$ times.

Let $3 r / 4-1<a_{n}<r+1$. We choose $\xi \in C_{n}$ such that $f(\xi)=1$. We denote $q_{n}=n\left(a_{n}+1\right)-n\left(a_{n}-1\right)$; here $q_{n} \geq 1$ because $C_{n}$ contains at least one zero of $f$. It is seen that $|1-\xi| t_{v} \mid<1$ for $\left|t_{\nu}\right| \geq 5 \mathrm{r} / 8$, and therefore

$$
0=\log |f(\xi)| \leq n(5 r / 8) \log r+q_{n} \log r_{n} .
$$

Now it follows from (b) that

$$
q_{n} \leq \frac{n(5 r / 8) \log r}{\log \left(1 / r_{n}\right)} \leq \frac{2 n(5 r / 8)}{K(\log r)^{1+\alpha}} .
$$

Let $b_{r}$ be the number of the points $a_{n}$ satisfying the condition $3 r / 4-1<a_{n}<r+1$. It follows from (a) that $b_{r} \leq \varepsilon^{-1}(\log r)^{\alpha}$, and we see from (1) that

$$
n(r)-n(3 r / 4) \leq \frac{2 n(3 r / 4)}{\varepsilon K \log r} .
$$


Therefore $n(r) \leq n(3 r / 4)(1+1 /(10 \log r))$ for all large values of $r$, and we get

$$
n(r) \leq n\left((3 / 4)^{4} r\right)\left(1+\frac{1}{5 \log r}\right)^{4} \leq n(r / e)\left(1+\frac{1}{\log r}\right) .
$$

Now it is seen that

$$
n\left(e^{m} r\right) \leq n(r) \prod_{i=1}^{m}\left(1+\frac{1}{t+\log r}\right) \leq n(r)(m+1) .
$$

This implies that $n(R)=O(\log R)$ as $R \rightarrow \infty$ and so $q_{n}=0$ for all large $n$. We are led to a contradiction and conclude that there exists an infinite subsequence $C_{n_{k}}$ of the discs $C_{n}$ such that $\{0,1\} \leftarrow f\left(C_{n_{k}}\right)$.

6. If $r$ is large, then $|1+r| t_{m} \mid>1$ for every $m$ and $|1+r| t_{m} \mid \geq 7 / 4$ for $m=1,2, \ldots, n(r)$. Therefore we obtain

and see that

$$
\log |f(-r)| \geq \log \left|b \prod_{m=1}^{n(r)}\left(1+r / t_{m}\right)\right| \geq n(r) \log (3 / 2),
$$

$$
n(r) \leq K_{1} \log M(r)
$$

for all large values of $r$.

We choose $n$ such that $\{0,1\} \notin f\left(C_{n}\right)$, and denote

$$
\delta_{n}=\frac{\varepsilon a_{n}}{2\left(\log a_{n}\right)^{a}} .
$$

If $|f(z)| \geq 3$ for every $z \in \Gamma_{n}=\left\{z:\left|z-a_{n}\right|=\delta_{n}\right\}$, then it follows from Rouche's theorem that $\{0,1\} \subset f\left(C_{n}\right)$ because $\left.f\left(C_{n}\right) \cap\{0,1)\right\} \neq \phi$. Therefore, there exists $\xi \in \Gamma_{n}$ such that $|f(\zeta)| \leq 3$, and we deduce from Schottky's theorem that $\log ^{+}|f(z)| \leq K_{2}$ on $\Gamma_{n}$. Applying Schottky's theorem in $U\left(a_{n}+i a_{n}, a_{n}-\delta_{n} / 2\right)$, we get $\log ^{+}\left|f\left(a_{n}+i a_{n}\right)\right| \leq$ $K_{3}\left(\log a_{n}\right)^{\alpha}$, and because $|f(z)| \leq f(-2|z|)$ if $|z|$ is large, it follows from Schottky's theorem that

$$
\log M\left(4 a_{n}\right) \leq K_{4}\left(\log a_{n}\right)^{a}
$$

if $n$ is large enough, say $n \geq n_{1}$, and $\{0,1\} \notin f\left(C_{n}\right)$.

If possible, we choose $n>n_{1}$ such that $\{0,1\} \notin f\left(C_{n}\right)$ and $\{0,1\} \subset$ $f\left(C_{n+1}\right)$, and $z_{1}, z_{2} \in C_{n+1}$ such that $f\left(z_{1}\right)=0$ and $f\left(z_{2}\right)=1$. We have $\left|z_{1}-z_{2}\right|<2 r_{n+1}$ and $\left|1-z_{2} / t_{m}\right|<1$ for $m>n\left(a_{n}+1\right)$. Therefore we obtain

$$
\begin{gathered}
0=\log \left|f\left(z_{2}\right)\right| \leq \log r_{n+1}+\log \left|b \prod_{m=1}^{n\left(a_{n}+1\right)}\left(1-z_{2} \mid t_{m}\right)\right| \\
<\log r_{n+1}+n\left(a_{n}+1\right) \log a_{n+1},
\end{gathered}
$$

and it follows from (b) that 


$$
n\left(a_{n}+1\right)>K\left(\log a_{n+1}\right)^{1+\alpha} .
$$

On the other hand, it follows from (2) and (3) that $n\left(a_{n}+1\right) \leq$ $K_{1} K_{4}\left(\log a_{n}\right)^{\alpha}$. This is in contradiction with (4), and we see that there exists $n_{2}>n_{1}$ such that $\{0,1\} \notin f\left(C_{n}\right)$ if $n \geq n_{2}$.

Let now $a_{n}-1 \leq r<a_{n+1}-1$. Then $n(r) \leq n\left(a_{n}+1\right)$ and we see from (2) and (3) that

$$
n(r)<K_{5}(\log r)^{\alpha}
$$

for all large values of $r$.

7. We denote $z_{n}=a_{n}+\left(a_{n}+i a_{n}\right) / 4$ and $s_{n}=a_{n}-\delta_{n}$. It follows from Schottky's theorem that $\log \left|f\left(z_{n}\right)\right| \geq 2 K_{6} \log M\left(a_{n}\right)$ where $K_{6}>0$. Because $s_{n} \in \Gamma_{n}$, we have $\log ^{+}\left|f\left(s_{n}\right)\right| \leq K_{2}$, and so

$$
\log \left|\frac{f\left(z_{n}\right)}{f\left(s_{n}\right)}\right| \geq K_{6} \log M\left(a_{n}\right) .
$$

Further, $\left|\left(t_{m}-z_{n}\right) /\left(t_{m}-s_{n}\right)\right|<1$ for $m>n\left(3 a_{n}\right)$, and therefore

$$
\log \left|\frac{f\left(z_{n}\right)}{f\left(s_{n}\right)}\right| \leq \log \prod_{m=1}^{n\left(3 a_{n}\right)}\left|\frac{t_{m}-z_{n}}{t_{m}-s_{n}}\right| \leq n\left(3 a_{n}\right) \log \left(6 a_{n} / \delta_{n}\right) .
$$

Because $\delta_{n}=\frac{\varepsilon a_{n}}{2\left(\log a_{n}\right)^{a}}$, we obtain

$$
\log \left|\frac{f\left(z_{n}\right)}{f\left(s_{n}\right)}\right| \leq K_{7} n\left(3 a_{n}\right) \log \log a_{n}
$$

and we see from (6) that for all sufficiently large $n$,

$$
n\left(3 a_{n}\right) \geq \frac{K_{8} \log M\left(a_{n}\right)}{\log \log a_{n}}
$$

where $K_{8}>0$.

It follows from $(5)$ and $(7)$ that $n(r)=O\left((\log r)^{2}\right)$ as $r \rightarrow \infty$ and $n(r) \neq O\left((\log r)^{d}\right)$ if $d<1$. Therefore we can choose $d, \quad 1 \leq d \leq 2$, such that $n(r)=O\left((\log r)^{d}\right)$ and $n(r) \neq O\left((\log r)^{d-\frac{1}{2}}\right)$. Then $\log M(r) \neq$ $O\left((\log r)^{d+\frac{1}{2}}\right)$ as $r \rightarrow \infty$.

Let us suppose that $a_{n+1}<a_{n}^{i}$ for all large $n$. Let $a_{n-1}<r \leq a_{n}$. Then it is seen from (7) that

$$
\log M(r) \leq \log M\left(a_{n}\right) \leq 2 K_{8}^{-1} n\left(r^{8}\right) \log \log r,
$$

and hence $\log M(r)=O\left((\log r)^{d+\frac{1}{4}}\right)$. We are led to a contradiction and conclude that there exist arbitrarily large values of $n$ such that $a_{n+1} \geq a_{n}^{7}$. 
8. We choose $n$ such that $a_{n+1} \geq a_{n}^{7}$ and set $g(z)=f\left(a_{n}+z^{2}\right)$. Because $a_{n}+\delta_{n} \in \Gamma_{n}$, we see that $\log ^{+}\left|g\left(\delta_{\frac{1}{n}}^{\frac{1}{n}}\right)\right| \leq K_{2}$. Applying Schottky's theorem in

we get

$$
U\left(a_{n}^{\frac{1}{2}} / 4, a_{n}^{\frac{1}{2}} / 4-\delta_{n}^{\frac{1}{2}} / 2\right),
$$

$$
\log \left|g\left(a_{n}^{\frac{1}{2}} / 4\right)\right| \leq K_{9}\left(\log a_{n}\right)^{\alpha / 2},
$$

and therefore $\log ^{+}\left|f\left(a_{n}+a_{n} / 16\right)\right| \leq K_{9} \log a_{n}$. Because $f$ omits the values 0 and 1 in $33 a_{n} / 32<|z|<35 a_{n} / 32$, we now see from Schottky's theorem that

$$
\log M\left(a_{n}+a_{n} / 16\right) \leq K_{10} \log a_{n} .
$$

This implies that

$$
\liminf _{r \rightarrow \infty} \frac{\log M(r)}{\log r}<\infty
$$

and we are led to a contradiction. Theorem 2 is proved.

\section{Proof of Theorem 3}

9. Let $0<a \leq 2$. We set $t=1 /(1+\alpha), a_{n}=e^{n^{t}}$ and $f(z)=$ $\prod_{m=1}^{\infty}\left(1-z / a_{m}\right)$. It is easily seen that

$$
a_{n+1}>a_{n}+\frac{a_{n}}{7\left(\log a_{n}\right)^{\alpha}}
$$

for all large $n$. Let $n>100$ and $z \in U\left(a_{n}, d_{n}\right)$ where

$$
d_{n}=\frac{a_{n}}{14\left(\log a_{n}\right)^{a}} .
$$

We choose positive integers $k$ and $p$ such that $a_{k-1}<a_{n} / 4 \leq a_{k}$ and $a_{p-1}<3 a_{n} \leq a_{p}$. Set

$$
f(z)=H(z) Q(z)\left(1-z / a_{n}\right) S(z)
$$

where $H(z)=\prod_{m=1}^{k-1}\left(1-z / a_{m}\right), \quad S(z)=\prod_{m=p}^{\infty}\left(1-z / a_{m}\right)$ and

$$
Q(z)=\left(1-z / a_{n}\right)^{-1} \prod_{m=k}^{p-1}\left(1-z / a_{m}\right) .
$$

We have

$$
\log |H(z)| \geq \log \prod_{m=1}^{k-1} \frac{a_{n}}{2 a_{m}} \geq(k-1) \log \left(a_{n} / 2\right)-\sum_{m=1}^{k-1} m^{t}
$$

and so $\log |H(z)| \geq n^{t+1 / 6}$. It follows that $\log |Q(z)|>-n$ and $\log |S(z)|>-n$. Therefore, 


$$
\log |H(z) Q(z) S(z)| \geq n^{t+1 / 7}
$$

in $U\left(a_{n}, d_{n}\right)$. Now we see that $|f(z)| \geq 2$ on $\left|z-a_{n}\right|=d_{n}$, and $f$ has therefore exactly one 1-point in $U\left(a_{n}, d_{n}\right)$. Let us denote by $\zeta$ this l-point of $f$. Because $f$ takes on the segment $\left[a_{n}-d_{n}, a_{n}+d_{n}\right]$ every value $w$ satisfying $-2 \leq w \leq 2$, we see that $\zeta$ lies on the real axis. It follows from (5) that

$$
\log \left|\frac{a_{n}}{\zeta-a_{n}}\right| \geq n^{t+1 / 7}
$$

and hence $\log \left|\zeta-a_{n}\right|^{-1}>n^{t+1} / 8=\left(\log a_{n}\right)^{2+\alpha} / 8$. Let $I_{n}$ denote the segment $\left(a_{n}-r_{n}, a_{n}+r_{n}\right)$ where

$$
\log \left(1 / r_{n}\right)=\left(\log a_{n}\right)^{2+\alpha} / 8
$$

Then $f$ has only a finite number of 1-points outside $U I_{n}$ and we see that $\cup I_{n}$ is not a Picard set for entire functions. Theorem 3 is proved.

\section{Proof of Theorem 5}

10. We construct the desired counterexamples with the aid of MittagLeffler's function

$$
E_{a}(z)=\sum_{n=0}^{\infty} \frac{z^{n}}{\Gamma(1+\alpha n)}
$$

where $0<a<2, E_{a}$ is of order $1 / \alpha, E_{\alpha}$ is bounded on $S(-\alpha \pi / 2$, $2 \pi-\alpha \pi / 2)$ and $\alpha E_{\alpha}(z)-\exp \left(z^{1 / \alpha}\right)$ is bounded on $S(-\alpha \pi / 2, \alpha \pi / 2)$.

Set $\alpha=1 / \varrho$. Let us suppose that there exist $L_{\beta}, L_{\gamma} \in J$ such that $\gamma=\beta+\alpha \pi$. We may suppose that $\beta=-\alpha \pi / 2$. We choose a slowly growing entire function $h$ such that $J_{h}=J$ and set $f(z)=h(z)\left(E_{\alpha}(z)+M\right)$, where $M>0$ is chosen such that $\left|E_{a}(z)\right|<M-1$ outside $S(-\alpha \pi / 2$, $\alpha \pi / 2)$. Then $J_{f}=J$ and $f$ is of order $\varrho$.

Let $1 / 2<\varrho<1$ and $\alpha=1 / \varrho$. If $\gamma \neq \beta+\alpha \pi$ for every $L_{\beta}, L_{\gamma} \in J$, then it follows from (i) that there exist $L_{\beta}, L_{\gamma_{s}} \in J$ such that $2 \pi-\alpha \pi<$ $\gamma-\beta \leq \pi$. We may assume that $\beta=-\gamma$ where $\pi-\alpha \pi / 2<\gamma \leq \pi / 2$. Set

with

$$
g(z)=E_{a}(z)+E_{\alpha}\left(-t^{\alpha} z\right)
$$

$$
t=\frac{\cos (\gamma \varrho)}{\cos (\gamma \varrho-\varrho \pi)} .
$$

Let $z=r e^{i \psi} \in S(\pi-\alpha \pi / 2, \alpha \pi / 2)$. Then

and

$$
\log ^{+}\left|E_{\alpha}(z)\right|=R_{1}(z)+r^{\varrho} \cos (\psi \varrho)
$$




$$
\log \left|E_{\alpha}\left(-t^{\alpha} z\right)\right|=R_{2}(z)+t r^{\circ} \cos (\psi \varrho-\varrho \pi),
$$

where $R_{1}(z)$ and $R_{2}(z)$ are bounded functions defined on $S(\pi-\alpha \pi / 2$, $\alpha \pi / 2)$. Now we see easily that for every $\varepsilon>0,|g(z)|$ tends to infinity uniformly, in $S(\beta+\varepsilon, \gamma-\varepsilon) \cup S(\gamma+\varepsilon, 2 \pi-\gamma-\varepsilon)$ as $|z| \rightarrow \infty$. If $h$ is a slowly growing function such that $J_{h}=J$, then $f(z)=h(z) g(z)$ satisfies $J_{f}=J$.

Let now $1 \leq \varrho<\infty$ and $\alpha=1 / \varrho$. If $\gamma \neq \beta+\alpha \pi$ for every $L_{\beta}$, $L_{\gamma} \in J$, then by (ii) there exist $L_{\beta}, L_{\gamma}, L_{\psi} \in J$ such that $\beta<\gamma<\psi$, $\gamma-\beta<\alpha \pi, \quad \psi-\gamma<\alpha \pi$ and $\psi-\beta>\alpha \pi$. We may assume that $\beta=-\alpha \pi / 2$. Set

$$
g(z)=E_{\alpha}(z)+E_{\alpha}\left(t^{\alpha} z e^{-i \varphi}\right)
$$

where $\varphi=\psi-\alpha \pi / 2$ and

$$
t=\frac{\cos (\gamma \varrho)}{\cos (\psi \varrho-\gamma \varrho-\pi / 2)} .
$$

Then $g(z)$ is bounded on $S(\psi, 2 \pi+\beta)$ and, for every $\varepsilon>0,|g(z)|$ tends to infinity uniformly on $S(\beta+\varepsilon, \gamma-\varepsilon) \cup S(\gamma+\varepsilon, \psi-\varepsilon)$ as $|z| \rightarrow \infty$. If $h$ is a slowly growing function such that $J_{h}=J$, then

$$
f(z)=h(z)(g(z)+M)
$$

satisfies $J_{f}=J$, provided the constant $M$ is chosen sufficiently large. Theorem 5 is proved.

\section{Proof of Theorem 4}

11. Let $E$ be an infinite closed set. We choose a linear mapping $L$ such that $\{0, \infty\} \subset F=L(E)$ and 0 is a limit point of $F$. Then $F$ contains an infinite countable set $A=\left\{a_{n}\right\}$ such that $\left|a_{1}\right|<1 / 4$ and $\left|a_{n+1}\right|<\left|a_{n}\right|^{5}$ for $n \geq 1$. We set

$$
f(z)=\prod_{n=1}^{\infty}\left(\frac{z-a_{n}}{z+a_{n}}\right) .
$$

In order to prove Theorem 4 , it is sufficient to prove that $f$ is normal in the complement of $B=A \cup\{0, \infty\}$. If $f$ is normal in $-B$, then it is normal in $-F$, and because both $\varrho(f(z))|d z|$ and $d \sigma_{G}(z)$ are conformal invariants, it follows from Theorem A that $f(L(\zeta))$ is normal in $-E$.

It follows from Theorem 1 of Lehto [6] that

$$
\limsup _{z \rightarrow 0}|z| \varrho(f(z))=\frac{1}{2} .
$$


Therefore there exists $M_{1} \geq 1 / 2$ such that

$$
\varrho(f(z)) \leq \frac{M_{1}}{|z|}
$$

for all $z$ satisfying the condition $0<|z| \leq 10$. Differentiation yields

$$
\varrho(f(z))=\frac{|f(z)|}{1+|f(z)|^{2}}\left|\sum_{k=1}^{\infty} \frac{2 a_{k}}{z^{2}-a_{k}^{2}}\right| .
$$

Because $\left|a_{1}\right|<1 / 4$ and $\left|a_{n+1}\right|<\left|a_{n}\right|^{5}$, we see easily that

$$
\varrho(f(z)) \leq \frac{4\left|a_{1}\right|}{|z|^{2}}
$$

in $|z| \geq 2\left|a_{1}\right|$, and

$$
\varrho\left(f(z) \leq \frac{4}{\left|a_{n}\right|}+\frac{4\left|a_{n+1}\right|}{|z|^{2}}\right.
$$

in $2\left|a_{n+1}\right| \leq|z| \leq\left|a_{n}\right| / 2$ for any $n \geq 1$.

We denote by $D$ the complement of the points 0,1 and $\infty$, and let $\sigma_{D}\left(w, w^{\prime}\right)$ be the hyperbolic metric of $D$. Constantinescu [4] has proved that

$$
\lim _{w \rightarrow 0}|w|\left(\log \left|\frac{1}{w}\right|\right) \frac{d \sigma_{D}(w)}{|d w|}=\frac{1}{2}
$$

Therefore there exists $\delta, 0<\delta \leq 1 / 2$, such that

$$
\frac{|d w|}{d \sigma_{D}(w)} \leq 4|w| \log \left|\frac{1}{w}\right|
$$

in $0<|w| \leq \delta$. The transformation $w=1 / z$ defines a conformal mapping of $D$ onto itself. Therefore $d \sigma_{D}(w)=d \sigma_{D}(z)$, and if $0<z \leq \delta$, we get from $(5)$

$$
\frac{|d w|}{d \sigma_{D}(w)}=\frac{|d z|}{|z|^{2} d \sigma_{D}(z)} \leq \frac{4}{|z|} \log \left|\frac{1}{z}\right|=4|w| \log |w|
$$

This implies that

$$
\frac{|d w|}{d \sigma_{D}(w)} \leq 4|w| \log |w|
$$

in $1 / \delta \leq|w|<\infty$. Similarly, we see by means of a linear transformation that there exists $M_{2}>0$ such that

$$
\frac{|d w|}{d \sigma_{D}(w)}<M_{2}
$$

in $0<|w-1| \leq \delta$. Because the set 


$$
T=\{z:|z| \geq \delta,|z-1| \geq \delta,|z| \leq 1 / \delta\}
$$

is a compact subset of $D$, there exists $M_{3} \geq M_{2}$ such that

$$
\frac{|d w|}{d \sigma_{D}(w)}<M_{3}
$$

in $T$.

We denote by $G$ the complement of $B=A \cup\{0, \infty\}$ and $D_{n}$ in the complement of the points $0, a_{n}$ and $\infty$. Because $D_{n} \supset G$, then $d \sigma_{D_{n}}(z) \leq d \sigma_{G}(z)$. Since $D$ is mapped conformally onto $D_{n}$ by $z=a_{n} w$, we have $d \sigma_{D_{n}}(z)=d \sigma_{D}(w)$, and therefore

$$
\frac{|d z|}{d \sigma_{G}(z)} \leq \frac{|d z|}{d \sigma_{D_{n}}(z)}=\frac{\left|a_{n}\right||d w|}{d \sigma_{D}(w)}
$$

We denote $Q_{1}=\left\{z:\left|a_{1}\right| / \delta \leq|z|<\infty\right\}$, and set for $n \geq 2$

$$
Q_{n}=\left\{z:\left|a_{n}\right| / \delta \leq|z| \leq\left|a_{n} a_{n-1}\right|^{\frac{1}{2}}\right\} \text {. }
$$

It follows from (9) and (6) that

$$
\frac{|d z|}{d \sigma_{G}(z)} \leq 4|z| \log \left|\frac{z}{a_{n}}\right|
$$

in $Q_{n}$. Let $z \in Q_{n}$. If $n=1$ we get from (10) and (3)

$$
\varrho(f(z)) \frac{|d z|}{d \sigma_{G}(z)} \leq 16\left|\frac{a_{1}}{z}\right| \log \left|\frac{z}{a_{1}}\right| \leq 8 .
$$

Let $n \geq 2$. It follows from (4) that

$$
\varrho(f(z)) \leq \frac{4}{\left|a_{n-1}\right|}+\frac{4\left|a_{n}\right|}{|z|^{2}}
$$

in $Q_{n}$ and we obtain from (10)

$$
\begin{gathered}
\varrho(f(z)) \frac{|d z|}{d \sigma_{G}(z)} \leq 16\left|\frac{a_{n}}{z}\right| \log \left|\frac{z}{a_{n}}\right|+16\left|\frac{z}{a_{n-1}}\right| \log \left|\frac{z}{a_{n}}\right| \\
\leq 8+\left.16\left|a_{n}\right| a_{n-1}\right|^{\frac{1}{2}} \log \left|a_{n-1} / a_{n}\right|^{\frac{1}{2}} \leq 16 .
\end{gathered}
$$

Let $\delta\left|a_{n}\right| \leq|z| \leq\left|a_{n}\right| / \delta$ and $z \in G$. It follows from (9), (7) and (8) that

$$
\frac{|d z|}{d \sigma_{G}(z)} \leq M_{3}\left|a_{n}\right|
$$

and we obtain from (2) and (3)

$$
\varrho(f(z)) \frac{|d z|}{d \sigma_{G}(z)} \leq \frac{M_{1} M_{3}\left|a_{n}\right|}{|z|} \leq \frac{M_{1} M_{3}}{\delta}=M_{4} .
$$

Let $\left|a_{n+1} a_{n}\right|^{\frac{1}{2}} \leq|z| \leq \delta\left|a_{n}\right|$. We get from (9) and (5)

$$
\frac{|d z|}{d \sigma_{G}(z)} \leq 4|z| \log \left|\frac{a_{n}}{z}\right|
$$


Together with (4) this implies that

$$
\begin{gathered}
\varrho(f(z)) \frac{|d z|}{d \sigma_{G}(z)} \leq 16\left|\frac{z}{a_{n}}\right| \log \left|\frac{a_{n}}{z}\right|+16\left|\frac{a_{n+1}}{z}\right| \log \left|\frac{a_{n}}{z}\right| \\
\leq 8+\left.16\left|a_{n+1} / a_{n}\right|^{\frac{1}{2}} \log \left|a_{n}\right| a_{n+1}\right|^{\frac{1}{2}} \leq 16 .
\end{gathered}
$$

We have proved that

$$
\varrho(f(z))|d z| \leq\left(M_{4}+16\right) d \sigma_{G}(z)
$$

for any $z \in G$. It follows from Theorem A that $f$ is normal in $G$. Theorem 4 is proved.

\section{References}

[1] Anderson, J. M., and J. Clunie: Entire functions of finite order and lines of Julia. - Math. Z. 112, 1969, 59-73.

[2] Baker, I. N., and L. S. O. Liverpool: Picard sets for entire functions. - Math. Z. $126,1972,230-238$.

[3] Cartwright, M. L.: Integral functions. - Cambridge Tracts in Mathematics and Mathematical Physics No. 44, Cambridge University Press, Cambridge, 1965 .

[4] Constantinescu, C.: Einige Anwendungen des hyperbolischen Masses. - Math. Nachr. 15, 1956, 155-172.

[5] Lehto, O.: A generalization of Picard's theorem. - Ark. Mat. 3, 1958, 495-500.

[6] - - The spherical derivative of meromorphic functions in the neighbourhood of an isolated singularity. - Comment. Math. Helv. 33, 1959, 196-205.

[7] - - , and K. I. VirTANen: Boundary behaviour and normal meromorphic functions. - Acta Math. 97, 1957, 47-65.

[8] Toppita, S.: Some remarks on the value distribution of meromorphic functions. Ark. Mat. 9, 1971, 1-9.

[9] - - - Some remarks on linear Picard sets. - Ann. Acad. Sci. Fenn. Ser. A I 569, $1973,1-17$.

[10] - - - On the value distribution of integral functions. - Ann. Acad. Sci. Fenn. Ser. A I 574, 1974, 1-20.

[11] Winkler, J.: Über Picardmengen ganzer und meromorpher Funktionen. - Math. Z. 109, 1969, 191-204.

University of Helsinki

Department of Mathematics

SF-00100 Helsinki 10

Finland

Received 18 November 1974 